\section{Revista de Salud Pública}

Journal of Public Health
Revista de Salud Pública

ISSN: 0124-0064

revistasp_fmbog@unal.edu.co

Universidad Nacional de Colombia

Colombia

Lira-García, Cynthia de; Souto-Gallardo, María; Bacardí-Gascón, Monserrat; Jiménez-Cruz, Arturo Revisión de la Efectividad de los Ingredientes de Productos Alternativos para la Pérdida de Peso

Revista de Salud Pública, vol. 10, núm. 5, diciembre, 2008, pp. 818-830

Universidad Nacional de Colombia

Bogotá, Colombia

Disponible en: http://www.redalyc.org/articulo.oa?id=42210514

- Cómo citar el artículo

- Número completo

- Más información del artículo

Página de la revista en redalyc.org

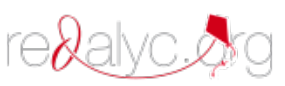

Sistema de Información Científica

Red de Revistas Científicas de América Latina, el Caribe, España y Portugal

Proyecto académico sin fines de lucro, desarrollado bajo la iniciativa de acceso abierto 


\section{Revisión de la Efectividad de los Ingredientes de Productos Alternativos para la Pérdida de Peso}

\section{A systematic review of the effectiveness of alternative weight-loss products' ingredients}

Cynthia de Lira-García, María Souto-Gallardo, Monserrat Bacardí-Gascón y Arturo Jiménez-Cruz

Universidad Autónoma de Baja California. Tijuana, B.C. México. ajimenez@uabc.mx

Recibido 4 Junio 2008/Enviado para Modificación 12 Octubre 2008/Aceptado 9 Noviembre 2008

\section{RESUMEN}

Objetivo La alta demanda para el tratamiento de la obesidad y la poca efectividad de las terapias convencionales favorecen el consumo de productos alternativos. Evaluar ensayos clínicos aleatorios sobre la pérdida de peso con los ingredientes de los productos alternativos de mayor consumo en México.

Metodología Se realizó una encuesta para valorar la prevalencia de consumo de productos alternativos para la pérdida de peso en Ensenada, Baja California. Se investigaron los productos más vendidos y se obtuvieron los componentes activos. Se revisaron ensayos clínicos aleatorios registrados en Pubmed para los componentes activos mas utilizados. Se incluyeron y analizaron aquéllos que evaluaran cambios en el peso.

Resultados Treinta y seis porciento de los encuestados han utilizado algún método alternativo para la pérdida de peso (83\% mujeres). Los productos más referidos fueron licuados, tés, pastillas y linaza. Se encontraron 16 ensayos clínicos aleatorios de cuatro ingredientes: Garcinia Cambodia, té verde, L-carnitina y linaza. Solamente dos estudios demostraron una pérdida de peso significativa $(2-5 \%)$ entre el grupo control y el experimental. En los estudios de L-Carnitina y linaza no se encontró una diferencia significativa.

Conclusión Se concluye que no hay evidencias suficientes y de calidad que justifiquen la utilización de los productos alternativos para la pérdida de peso.

Palabras Clave: Pérdida de peso, suplementos dietéticos, terapias complementarias, obesidad (fuente: DeCS, BIREME).

\section{ABSTRACT}

Objective The high demand for obesity treatment and conventional therapies' lack of effectiveness increases the use of alternative products. This study was aimed at 
assessing evidence from randomised clinical trials regarding the effectiveness of alternative weight-loss products' ingredients.

Methods A survey was conducted in Ensenada, Baja California, to assess the frequency of consuming alternative products used for weight reduction. The ingredients in the products most in demand were included in this review. The Pubmed database was searched for all randomised clinical trial papers including these ingredients and assessing weight loss.

Results Thirty-six percent of the subjects questioned had used alternative methods for weight-loss, $83 \%$ of them being women. The most frequently used products were shakes, tea, pills and flaxseed/linseed. Sixteen randomised clinical trials using four ingredients were used: Garcinia cambogia, green tea, L-carnitina and flaxseed. Only two studies (green tea and Garcinia cambogia) showed significant weight-loss (2-5\%) amongst control and experimental groups. There was no significant difference between the groups in the studies regarding L-carnitina and flaxseed.

Conclusions There is a lack of evidence regarding quality studies justifying the use of alternative products for weight loss.

Key Words: Weight loss, dietary supplement, complementary therapy, obesity (source: $\mathrm{MeSH}, \mathrm{NLM})$.

$\mathbf{E}$ l sobrepeso y la obesidad en las últimas décadas se han incrementado drásticamente convirtiéndose en un problema de salud pública (1-6). Esta tendencia puede ser debida a factores genéticos, prenatales, postnatales, y el consumo excesivo de alimentos hipercalóricos combinado con el sedentarismo (7-18). La obesidad se ha incrementado a tal magnitud, que ha rebasado los índices de la desnutrición (19). En el mundo, el sobrepeso está presente en más de mil millones de personas y una tercera parte de ellos se encuentra en obesidad clínica. Según datos de la Organización Mundial de la Salud (OMS), el problema no sólo afecta a la población adulta, sino también a los menores tanto de países desarrollados como en vías de desarrollo (20). De acuerdo a la Encuesta Nacional de Salud y Nutrición (ENSANUT 2006), en México el $72 \%$ y el $67 \%$ de mujeres y hombres respectivamente, mayores de 20 años tienen un Índice de Masa Corporal $\geq 25 \mathrm{~kg} / \mathrm{m}^{2}$, y uno de cada tres adolescentes presentan sobrepeso u obesidad (19). Esto es relevante para la salud ya que es un factor de riesgo potencial para enfermedades crónicodegenerativas (9-12).

La efectividad de los programas convencionales para el tratamiento de la obesidad es inferior a las expectativas de quienes la padecen. Lo que aunado a la insuficiente regulación, control sanitario y a una falta de información adecuada para la población, ha favorecido el consumo de productos alternativos 
o "naturales” que se pueden obtener sin receta médica (21).

Existen revisiones de algunas sustancias naturales con resultados no concluyentes sobre su efecto en la pérdida de peso (22-24).

El objetivo de éste artículo es evaluar ensayos clínicos aleatorios (ECA) sobre la pérdida de peso con los ingredientes de los productos alternativo de mayor consumo en México.

\section{METODOLOGÍA}

Durante los meses de Febrero y Marzo de 2008 se entrevistaron en la ciudad de Ensenada, Baja California a personas mayores de 16 años en varios centros de trabajo. Se registró la edad y el sexo, el antecedente del consumo de productos alternativos (hierbas, tés, suplementos, medicamentos "naturales", licuados, etc) para la pérdida de peso y el nombre del producto. Se estimaron los productos de mayor consumo. Posteriormente se entrevistaron a los responsables de 8 establecimientos dedicados a la venta de estos productos ubicados en la zona centro y zonas comerciales de la ciudad de Ensenada, Baja California (Farmacias Similares, GNC, Siluet 7, Costco, Comercial Mexicana, Walmart y 3 tiendas de productos naturales y hierbas). Se identificaron los productos más vendidos y se obtuvieron de las etiquetas los componentes activos.

Se revisaron todos los artículos originales de ECA registrados en Pubmed publicados del 1 de Enero de 2000 al 31 de Enero de 2008 con los componentes activos de consumo más frecuentes. Se utilizaron las siguientes palabras clave: "pingüica, uva ursi, arctostaphylos uva ursi, cocolmeca, Garcinia cambodia, hydroxycitric acid, L-carnitine, linseed, linum usitatissimum, lecithin, phosphatidyl choline, green tea, nopal, nopal pad, kelp, laminaria, apple cider vinegar, tlanchalagua, senna, Cassia agustifolia" y combinaciones con "weight" y "weight loss". Se seleccionaron los trabajos que incluían resúmenes completos y evaluaran cambios en el peso. Se analizaron los artículos seleccionados y sus referencias.

De cada artículo se revisó el diseño del estudio, la duración de la intervención, el número de participantes, el porcentaje de retención al final de la intervención, edad, género, índice de masa corporal (IMC), el poder estadístico del estudio, la reducción de peso en $\mathrm{kg}$, las diferencias de los resultados entre grupos y su nivel de significancia y los efectos adversos. Se valoró la calidad de los estudios incluidos aplicando la puntuación de Jadad (25) obteniéndose 
como calificación máxima 5 puntos para los estudios con mayor calidad. Cada artículo fue revisado de manera independiente por dos investigadores (CDL y MSG), cuando no hubo consistencia se consensuó de manera conjunta. Posteriormente fue revisado por otro investigador (AJC), y se repitió el proceso.

\section{RESULTADOS}

La encuesta fue aplicada a un total de 188 personas de ambos sexos (16 y 61 años). El $36 \%$ de ellos respondió haber utilizado algún método alternativo para la pérdida de peso (83\% mujeres) y de éstos el $43 \%$ eran menores de 20 años. Los productos más referidos fueron licuados (25\%), de los cuales los más frecuentes fueron los productos de Herbalife. En segundo los tés y pastillas con $20 \%$ cada uno; de los cuales los más mencionados fueron el té de tlanchalagua, el té rojo y el té verde (TeV). En tercer lugar la linaza con $5 \%$. Los productos más vendidos fueron: Linopal plus, Linopal Premium, Linaza, Body Slim, Tlanchalagua, Dieter's drink, Amino Slim, Simigras, Simisiluet, Simigen coffee, Simikelp, Similinaza, Lecitina de soya, Simicarnitina, Siluet 7, Demograss, Metaboltonics y Capslim. Los componentes activos incluídos con mayor frecuencia en esos productos fueron: pingüica (Arctostaphylos uva ursi), cocolmeca, ácido hidroxicítrico (Garcinia Cambogia), L-carnitina, linaza, lecitina de soya (fosfatidilcolina), TeV, extracto de nopal, Kelp (laminaria), vinagre de manzana, tlanchalagua y hoja de senn (Cassia angustifolia).

Se encontraron 16 ECA que cumplían los criterios de inclusión: 5 de Garcinia Cambogia (GC), 5 de TeV, 4 de L-carnitina y 2 de linaza. En la Tabla 1 se presenta la descripción de las características y resultados de estos estudios. No se encontraron ECA publicados de los demás productos.

Garcinia cambogia

El ácido hidroxicítrico (HCA) se obtiene del extracto de la GC. Se han publicado estudios que describen el efecto de esta substancia sobre la inhibición en la formación de acetil CoA y sobre la inhibición de lipogénesis de novo. Algunos autores sugieren que ese efecto podría aumentar la oxidación de lípidos y la saciedad (26-29).

Mattes realizó un estudio con 167 sujetos con sobrepeso durante 12 semanas. La intervención consistió en la ingesta de $1200 \mathrm{mg} / \mathrm{d}$ de HCA o placebo con una dieta de $1200 \mathrm{kcal}$. Los resultados mostraron una pérdida de peso aproximada del $5 \%$ y 3,4 \% del grupo de intervención y el grupo placebo 
respectivamente y con diferencia significativa entre los grupos $(\mathrm{P}=0,026)$.

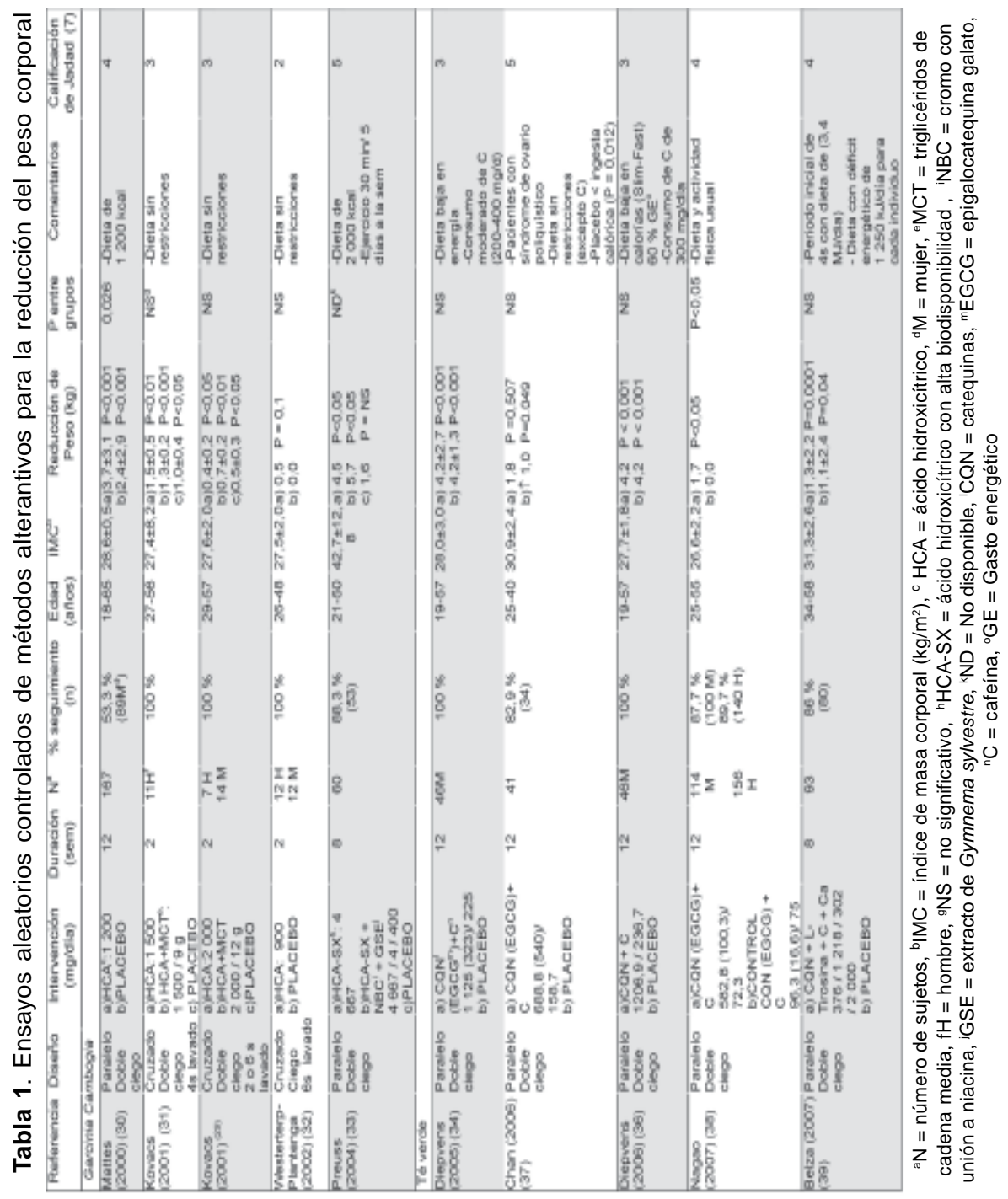


En este estudio hubo un porcentaje bajo de seguimiento (53\%) debido a que hubo un error de codificación de los tratamientos y eliminaron a 28 sujetos (30).

Tres estudios de Kovacs de 2 semanas de duración, no reportaron efecto significativo en la pérdida de peso entre el grupo con tratamiento (HCA) y el grupo control. El que reportó mayor pérdida fue de 1,7 \% para el grupo de HCA y $1,2 \%$ para el grupo placebo. Para ninguno de los estudios de este grupo se controló la dieta ni la actividad física; sin embargo, en dos se observó pérdida de peso tanto en el grupo de tratamiento como en el grupo control. Dos de esos estudios incluyeron un grupo con HCA y triglicéridos de cadena media $(29,31,32)$.

Preuss evaluó el efecto del HCA sobre la pérdida de peso en 60 sujetos con obesidad durante 8 semanas. Se observó una reducción significativa de peso al final de la intervención para cada grupo de $5 \%$ y $2 \%$ del grupo de tratamiento y control respectivamente. No reportaron si existía diferencia significativa entre los grupos (33).

Té verde

El TeV está hecho de hojas de Camellia sinensis y contiene grandes cantidades de cafeína y componentes polifenólicos, como la epicatequina (EG), la epigalocatequina (EGC), y la galato de epigalocatequina (EGCG) (34). Algunos autores sugieren que el $\mathrm{TeV}$ tiene un efecto sobre la pérdida de peso mediante un aumento de la termogénesis y de la oxidación de grasas $(35,36)$.

Se analizaron cuatro ensayos clínicos aleatorios de 12 semanas de intervención con catequinas. Dos realizados por Diepvens en 46 mujeres con sobrepeso donde a los grupos experimentales se les administraron dosis de más de $1000 \mathrm{mg} / \mathrm{d}$ de catequinas. A los dos grupos se les prescribió una dieta baja en calorías (Slim-Fast). En ambos grupos se observó una pérdida aproximada de 5,5 \% entre el inicio y el final de la intervención, pero la diferencia entre los grupos no fue estadísticamente significativa $(34,36)$.

En el tercer estudio elaborado por Chan participaron 41 mujeres con síndrome de ovario poliquístico y con un IMC promedio de $30,9 \pm 2,4 \mathrm{~kg} / \mathrm{m}^{2}$. Se les prescribió 689 mg/d de EGCG o placebo, sin restricción en la dieta. No se observó una pérdida significativa de peso al final de la intervención, ni diferen- 
cia entre grupos (37).

El cuarto estudio realizado por Nagao evaluó 270 sujetos con dosis de 583 mg/d de EGCG. Se observó una pérdida de peso de 2,3\% en el grupo experimental y $0 \%$ en el control y una diferencia significativa entre grupos $(\mathrm{P}<0,05)$. Este estudio tuvo una calificación de 4 pero no hubo control de actividad física y dieta (38).

Belza realizó un estudio de 8 semanas con 93 pacientes a los que se les suministró catequinas combinadas con tirosina y calcio $(1$ 218, 376, $2000 \mathrm{mg} /$ d) o placebo. Se observó una pérdida de peso de 1,5 \% y 1,4\% en el grupo de tratamiento y el control respectivamente, sin haber diferencia significativa entre grupos (39).

\section{L-Carnitina}

La L-carnitina interviene en la oxidación de ácidos grasos de cadena larga. Algunos autores han sugerido que ésta aumenta el rendimiento físico, lo que podría favorecer la pérdida de peso (40).

Hongu y Sachan realizaron un estudio de 5 semanas de duración en 19 individuos entre 18 y 54 años con un IMC entre 18,9 y 35,9 kg/m². Los dividieron en dos grupos pareados sin hacer cambios en la dieta o estilo de vida. En el grupo de intervención se dio L-carnitina + colina (680 / 940 mg/día), en el que se observó una reducción de $1.6 \mathrm{~kg}(\mathrm{P}<0,05)$, mientras que en el grupo placebo se observó reducción de $0,7 \mathrm{~kg}(\mathrm{p}<0,05)$. Este estudio no reporta la diferencia entre los grupos y tiene una calificación de 1 en la puntuación de Jadad (41).

Lofgren et al realizaron un estudio doble ciego en mujeres de 20 a 45 años, durante 10 semanas. La adherencia al final del tratamiento fue de $82 \%$. El tratamiento incluyó una dieta con restricción calórica, aumento de la actividad física y al grupo de intervención $3000 \mathrm{mg}$ /día de carnitina. No se observó pérdidas de peso significativas al final del estudio, ni diferencia significativa entre los dos grupos. La calificación de calidad de acuerdo a los criterios de Jadad fue de 3 (40).

Derosa et al también realizarion un estudio doble ciego durante 26 semanas. Participaron 47 hombres y 47 mujeres entre 45 y 57 años y con un IMC de $27,3 \pm 2,5 \mathrm{~kg} / \mathrm{m}^{2}$. La calidad del estudio fue de 4 de acuerdo a los criterios de Jadad. Se prescribió una dieta de 400 a 600 kcal/día para ambos grupos. El 
grupo de intervención recibió 2000 mg/día de L-carnitina. No se observaron pérdidas significativas de peso, ni diferencias entre grupos al final del estudio (42).

Elmslie et al estudiaron el efecto de la L-carnitina en 60 sujetos con obesidad (49 mujeres y 11 hombres. Se observó una pérdida de 1,9 kg y 0,9 kg en el grupo de tratamiento $(15 \mathrm{mg} / \mathrm{kg} /$ día de L-carnitina) y el control respectivamente. Sin embargo, esta pérdida no fue significativa ni se observó diferencia entre los dos grupos. Este estudio, con una evaluación de calidad de 5 (criterio de Jadad) se realizó durante 26 semanas e incluyó una restricción de 500 kcal en la dieta (43).

Linaza

Algunos autores atribuyen los beneficios de la linaza al contenido de ácido linoléico (LA) y ácido á-linolénico (ALA), que se han recomendado para disminuir las enfermedades cardiovasculares $(44,45)$. Sin embargo solamente se encontraron dos ensayos clínicos aleatorios relacionados con el cambio en el peso corporal.

Paschos et al realizaron una intervención de 12 semanas en 40 hombres con sobrepeso entre 38 y 71 años, en el que se incluyó $15 \mathrm{mg} /$ día de aceite de linaza (8,1 g ALA) y $15 \mathrm{mg} /$ día de aceite de cártamo (11,2 g LA). No se prescribieron cambios dietéticosa. Se observó un incremento de peso en el grupo tratado con ALA. Sin embargo los resultados no fueron significativos (45).

Schwab et al realizaron un estudio doble ciego durante cuatro semanas, en 16 sujetos ( 8 mujeres y 8 hombres) entre 25 y 60 años con peso normal y con sobrepeso. Se prescribió $30 \mathrm{mg} /$ día de aceite de linaza y $30 \mathrm{mg} /$ día de aceite de cártamo. Al final del estudio se observó un un incremento en el peso no significativo estadísticamente (46).

\section{DISCUSIÓN}

De los 16 artículos analizados solamente dos mostraron una reducción significativa de peso, uno de $G C$ y otro de $\mathrm{TeV}(30,38)$. Estos estudios tuvieron una duración de 12 semanas y una calificación alta (4 puntos) según la puntuación de Jadad (25). Sin embargo, el estudio de Mattes que evalúa el efecto de la GC presentó un porcentaje de retención bajo (53\%) (30). Se publicaron otros 
estudios antes del año 2000, los cuales reportaron resultados contradictorios (21-23). Se han publicado estudios que describen el efecto de la GC sobre la liberación de serotonina a nivel cerebral, lo que se ha considerado como el mecanismo principal para la supresión del apetito (47). En estudios recientes en ratas se ha observado el efecto del ácido hidroxicítrico sobre los genes reguladores de la obesidad y la lipogénesis de novo $(47,48)$.

De acuerdo a los ECA analizados del TeV, se puede concluir que no hay evidencias sobre su efectividad para la reducción de peso. Sin embargo, hay diversos estudios que describen efectos metabólicos importantes $(49,50)$. En una investigación in vitro se observó disminución de la digestión de grasa en el tracto gastrointestinal por el efecto inhibitorio del TeV sobre las lipasas (49). En otro estudio in vitro se observó que el $\mathrm{TeV}$ tiene mayor efectividad sobre la termogénesis en tejidos periféricos que dosis equivalentes de cafeína(50). Estos hallazgos fueron confirmados en estudios realizados en humanos. Se observó que estas diferencias no estuvieron asociadas a un incremento en la frecuencia cardiaca (50), lo que permitiría utilizarse en pacientes con hipertensión y enfermedad cardiaca.

De todos los artículos revisados solamente uno, que valora el efecto de la L-carnitina, describe el poder estadístico y los resultados no mostraron diferencia estadísticamente significativa sobre la pérdida de peso (43). Sin embargo existen estudios que muestran mejoría en el rendimiento físico (51).

Con respecto a los efectos adversos, solamente ocho estudios los evaluaron. En tres de ellos (GC y TV) no observaron diferencias significativas entre el grupo de tratamiento y el grupo control $(30,33,39)$. En los cinco estudios restantes no encontraron ningún efecto adverso (29,31,32,34,37).

Para valorar el impacto de los productos alternativos en la sociedad mexicana se buscaron los datos de la prevalencia del consumo de estos según sexo y edad. En México no se encontró una encuesta acerca del consumo de productos alternativos para bajar de peso, sin embargo consideramos que hay una tendencia similar a la de Estados Unidos, donde el uso de estos productos era cinco veces mayor en mujeres, principalmente en jóvenes con sobrepeso y obesidad, aunque el $8 \%$ tenían un peso normal (52). La encuesta que realizamos no es representativa de la población de Ensenada, sin embargo una tercera parte de personas entrevistadas referían haber utilizado productos alternativos para la pérdida de peso. Lo que sugiere que existe una alta demanda de este tipo de productos. Por lo que se hace necesario una mayor vigilancia de 
estos productos, debido a que no se han evaluado, mediante diseños adecuados, los efectos indeseables y se ofrecen expectativas no basadas en evidencias científicas. Recientemente los gobiernos de Estados Unidos, Canadá y México se han unido para formar el Grupo contra Fraudes a la Salud (MUCH) (2003), que tiene como propósito realizar acciones en contra de las empresas que promueven publicidad falsa y engañosa para bajar de peso y que además pueden poner en riesgo la salud de los consumidores (53). Esta es una medida importante para el control de estos productos “naturales” y debe ser apoyada con estudios sistemáticos de calidad.

Este estudio tiene como limitaciones el haber usado solamente como base de datos el Pubmed. Además, el hecho de que no es posible determinar los estudios realizados y no publicados.

Solamente un estudio de GC y uno de TV mostraron diferencias estadísticamente significativas, mediante una intervención a corto plazo, lo que permite concluir que no se ha podido comprobar la efectividad de los productos alternativos para la pérdida de peso

Agradecimientos. Los autores agradecen las becas otorgada por el CONACYT. No. Proj: 95407300449771.

Conflictos de Interés: Ninguno

\section{REFERENCIAS}

1. The World Health Organization warns of the rising threat of heart disease and stroke as overweight and obesity rapidly increase [internet]. Disponible en: http://www.who.int/ mediacentre/news/releases/2005/pr44/en/index.html. Consultado abril 2008.

2. World Health Organization. Obesity: Preventing and managing the global epidemic. Tech Report Serv 2000; 894:1-253.

3. Rivera JA, Barquera S, Campirano F, Campos I, Safdie M, Tovar V. Epidemiological and nutricional transition in México: rapid increase of non-communicable chronic diseases and obesity Public Health Nutrition 2002; 5(1A): 113-122.

4. Jimenez-Cruz A, Bacardi Gascon M. Prevalence of Overweight and Hunger among Mexican Children from Migrant Parents. Nutr Hosp 2007;22(1):85-8.

5. Jimenez-Cruz A, Bacardi-Gascon M, Jones E. Extreme Obesity among Children in Mexico. J Pediatrics 2007; 151(3):e12-e13.

6. Toussaint MG. Obesidad en niños. Bol Hosp Inf Mex 2007; 64: 347-348.

7. Comuzzie AG, Allison DB. The Search for Human Obesity Genes. Science 1999; 280. (5368): $1374-137$.

8. Proietto J. Why staying lean is not a matter of ethics. MJA 1999; 171: 611-613.

9. Dietz WH. Health consequences of overweight in youth: childhood predictors of adult disease. Pediatrics 1998; 101:518-525.

10. Dulloo AG, Jacquet J, Seydoux J, Montani JP. The thrifty 'catch-up fat' phenotype:its impact 
on insulin sensitivity during growth trajectories to obesity and metabolic syndrome. Int J Obes 2006; 30 (Supl. 2): S23-35.

11. Jiménez-Cruz A, Bacardi-Gascon $M$. The fattening burden of type 2 diabetes to Mexicans: projections from early growth to adulthood. Diabetes Care 2004;27(5): 1190-1193.

12. Aronne LJ, Isoldi KK. Overweight and obesity: key components of cardiometabolic risk. Clin Cornerstone 2007; 8(3): 29-37.

13. Hill JO, Sallis JF, Peters JC. Economic Analysis of Eating and Physical Activity. A Next Step for Research and Policy Change. Am J Prev Med 2000; 27 (3S):111-116.

14. Dixon HG, Scully ML, Wakefield MA, White VM, Crawford DA. The effect of television advertisements for junk food versus nutritious food on children's food attitudes and preferences. Soc Sci Med 2007; 65:1311-1323.

15. Robinsson T. Does television cause childhood obestity?. JAMA 1999; 279: 959-960.

16. Davison KK, Marshall SJ, Birch LL. Cross-sectional and longitudinal associations between television viewing and girls' body mass index, overweight status, and percentage of body fat. J Pediatr 2006;149 (1): 32-37.

17. Dietz WH, Gortmaker SL. Do we fatten our children at the television set? Obesity and television-viewing in children and adolescents. Pediatrics 1985;75(5): 807-812.

18. Gortmaker SL, Must A, Sobol AM, Peterson K, Colditz GA, Dietz WH. Television-viewing as a cause of increasing obesity among children in the United States, 1986-1990. Arch Pediatr Adolesc Med 1996;150:356-362.

19. Olaiz-Fernandez G, Rivera-Dommarco J, Shama-Levy T, Rojas R, Villalpando-Hernández S, Hernández-Ávila M, et al. Encuesta Nacional de Salud y Nutrición 2006. Cuernavaca, México: Instituto Nacional de Salud Pública; 2006.

20. Villa AR, Escobedo MH, Méndez-Sánchez N. Estimación y proyección de la prevalencia de obesidad en México a través de la mortalidad por enfermedades asociadas. Gac Méd Méx 2004; 140(2):S21-26.

21. Cummings S, Parham ES, Strain GW. Position of the American Dietetic Association: Weight management. J Am Diet Assoc 2002; 102(8): 1145-55.

22. Allison DB, Fontaine KR, Heshka S, Mentore JL, Heymsfield SB. Alternative treatment for weight loss: a critical review. Crit Rev Food Sci Nutr 2001; 41(1):1-28.

23. Pittler MX, Ernst E. Dietary supplements for body-weight reduction: a systematic review. Am J Clin Nutr 2004; 79: 529-536.

24. Saper RB, Eisenberg DM, Phillips RS. Common Dietary Supplements for Weight Loss. Am Fam Physician 2004; 70: 1731-38.

25. Jadad AR, Moore RA, Carroll D, Jenkinson C, Reynolds DJM, Gavaghan DJ, et al. Assessing the Quality of Reports of Randomized Clinical Trials: Is Blinding Necessary? Control Clin Trials 1996;17: 1-12.

26. McCarty MF. Promotion of hepatic lipid oxidation and gluconeogenesis as a strategy for appetite control. Med Hypotheses 1994; 42:214-225.

27. McGarry JD, Foster DW. In support of the roles of malonyl-CoA and carnitine acyltrasferase I in the regulation of hepatic fatty acid oxidation and ketogenesis. J Biol Chem 1979; 254:8163-8168.

28. Sullivan AC, Hamilton JG, Miller ON, Wheatley VR. Inhibition of lipogenesis in rat liver by (-)hydroxycitrate. Arch Biochem Biochim Pol 1976; 23:227-234.

29. Kovacs EMR, Westerterp-Plantenga MS, De Vries M, Brouns F, Saris WHM. Effects of 2week ingestion on (-)-hydroxycitrate and (-)-hydrocycitrate combined with mediumchain triglycerides on satiety and food intake. Physiol Behav 2001; 74: 543-549.

30. Mattes RD, Bormann L. Effects of (-)-hydroxycitric acid on appetitive variables. Physiol Behav 2000; 71: 87-94.

31. Kovacs EMR, Westerterp-Plantenga MS, Saris WHM. The effects of 2-week ingestion of ()-hydroxycitrate and (-)-hydroxycitrate combined with medium-chain triglycerides on satiety, fat oxidation, energy expenditure and body weight. Int J Obes 2001; 25:10871094. 
32. Westerterp-Planenga MS, Kovacs EMR. The effect of (-)-hydroxycitrate on energy intake and satiety in overweight humans. Int J Obes 2002; 26: 870-872.

33. Preuss HG, Bagchi D, Bagchi M, Rao CVS, Dey DK, Satyanarayana S. Effects of a natural extract of (-)-hydroxycitric acid (HCA-SX) and a combination of HCA-SX plus niacinbound chromium and Gymnema sylvestre on weight loss. Diabetes Obes Metab 2004; 6:171-180.

34. Diepvens K, Kovacs EMR, Nijs IMT, Vogels M, Westerterp-Plantenga MS. Effect of green tea on resting energy expenditure and substrate oxidation during weight loss in overweight females. Br J Nutr 2005; 94:1026-1034.

35. Dulloo AG, Duret C, Rohrer D, Girardier L, Mensi N, Fathi M, et al. Efficacy of green Tea extract rich in catechin polyphenols and caffeine in increasing 24-h energy expenditure and fat oxidation. Am J Clinic Nutr 1999; 70:1040-1045.

36. Diepvens K, Kovacs EMR, Vogels N, Westerterp-Plantenga MS. Metabolic effects of green tea and of phases of weight loss. Physiol Behav 2006; 87:185-191.

37. Chan CCW, Koo MWL, Ng EHY, Tang O, Yeung WSB, Ho P. Effects of Chinese green tea on weight, and hormonal and biochemical profiles in obese patients with polycystic ovary syndrome- A randomized placebo-controlled trial. J Soc Gynecol Investig 2006; 13:63-68.

38. Nagao T, Hase T, Tokimitsu I. A Green Tea Extract High in Catechins Reduces Body Fat and Cardiovascular Risks in Humans. Obesity 2007; 15:1473-1483.

39. Belza A, Frandsen E, Kondrup J. Body fat loss achieved by stimulation of termogénesis by a combination of bioactive food ingredients: a placebo-controlled, double-blind 8week intervention in obese subjects. Int J Obes 2007; 31:121-130.

40. Lofgren I, Zern T, Herron K, West K, Sharman MJ, Volek JS, et al. Weight loss associated with reduced intake of carbohydrate reduces the atherogenicity of LDL in premenopausal women. Metab Clin Exp 2005; 54:1133-1141.

41. Hongu N, Sachan DS. Carnitine, Choline Supplementation with Exercise Alter Carnitine Profiles, Biochemical Markers of Fat Metabolism and Serum Leptin Concentration in Healthy Women. J Nutr 2003; 133:84-89.

42. Derosa G, Cicero AFG, Gaddi A, Mugellini A, Ciccarelli L, Fogari R. The Effect of L-carnitine on plasma lipoprotein(a) Levels in Hypercholesterolemic Patients with Type 2 Diabetes Mellitus. Clin Ther 2003;25(5):1429-39.

43. Elmslie JL, Porter RJ, Joyce PR, Hunt PJ, Mann JI. Carnitine does not improve weight loss outcomes in valproate-treated bipolar patients consuming an energy-restricted, lowfat diet. Bipolar Disord 2006; 8:503-507.

44. Harper CR, Edwars MJ, DeFilipis AP, Jacobson TA. Flaxseed Oil Increases the Plasma Concentrations of Cardioprotective (n-3) Fatty Acids in Humans. J Nutr 2006;136:8387.

45. Paschos GK, Zampelas A, Panagiotakos DB, Katsiougiannis S, Griffin BA, Votteas V, et al. Effects of flaxseed on supplementantion on plasma adiponectin level in dyslipidemic men. Eur J Nutr 2007; 46:315-320.

46. Schwab US, Callaway JC, Erkkilä AT, Gynther J, Uusitupa MIJ, Järvinen T. Effects of hempseed and flaxseed oils on the profile of serum lipids, serum total and lipoprotein lipid concentrations and haemostatic factores. Eur J Nutr 2006; 45:470-477.

47. Downs BW, Bagchi M, Subbaraju GV, Shara MA, Preuss HG, Bagchi D. Bioefficacy of a novel calcium-potasium salt of (-)-hydroxycitric acid. Mutat Res 2005; 579:149-162.

48. Kovacs EMR, Westerterp-Plantenga MS. Effects of (-)-hydroxycitrate on net fat synthesis as de novo lipogenesis. Physiol Behav 2006; 88:371-381.

49. Juhel C, Armand M, Pafumi Y, Rosier C, Vandermander J, Lairon D. Green Tea extract (AR25) inhibits lipolisys of triclycerides in gastric and duodenal medium in vitro. J Nutr Biochem 2000; 11:45-51.

50. Dulloo AG, Seydoux J, Girardier L, Chantre P, Vandermander J. Green tea and thermogenesis: interactions between catechin-polyphenols, caffeine and sympathetic activity. Int J 
830 REVISTADE SALUD PÚBLICA·Volumen 10 (5), Diciembre 2008

obesity 2000; 24:252-258.

51. Hongu N, Sachan DS. Carnitine and Choline Supplementation with Exercise Alter Carnitine Profiles, Biochemical Markers of Fat Metabolism and Serum Leptin Concentration in Healthy Women.. J Nutr 2003; 133:84-89.

52. Blanck HM, Khan LK, Serdula MK. Use of Nonprescription Weight Loss Products. JAMA 2001;286:930-935.

53. México, Estados Unidos y Canadá trabajan conjuntamente contra los productos "Milagro" [Internet]. Disponible en: http://www.cofepris.gob.mx/bv/comunicados/b62.pdf. Consultado abril del 2008. 